\title{
GEOMETRIC INTERPRETATION OF THE RECURSION OPERATORS FOR THE GENERALIZED ZAKHAROV-SHABAT SYSTEM IN POLE GAUGE ON THE LIE ALGEBRA $A_{2}$
}

\author{
ALEXANDER B. YANOVSKI
}

Communicated by Metin Gürses

Abstract. We consider the recursion operator approach to the soliton equations related to a generalized Zakharov-Shabat auxiliary linear system in pole gauge on the Lie algebra $A_{2}=\mathfrak{s l}(3, \mathbb{C})$ and show that the recursion operator can be identified with the dual to a Nijenhuis tensor for a Poisson-Nijenhuis structure on the manifold of potentials.

\section{Introduction}

The soliton equations or completely integrable equations have been object of intense study since their discovery. Their most essential property is that they admit a Lax representation $[L, A]=0$. In it $L, A$ are linear operators on $\partial_{x}, \partial_{t}$ depending also on some functions $q_{i}(x, t), 1 \leq i \leq s$ ('potentials') and a spectral parameter $\lambda$. The equation $[L, A]=0$ should be satisfied identically in $\lambda$ and in this way the Lax equation $[L, A]=0$ is equivalent to a system of partial differential equations for $q_{i}(x, t)$. Usually one fixes the linear problem $L \psi=0$ (auxiliary linear problem) and considers all the evolution equations (of certain form of course) one can obtain changing the operator $A$. These equations are called nonlinear evolution equations (NLEEs) associated (related) with $L$ (or with the linear system $L \psi=0$ ). There are several different schemes to resolve them but the essential point is that the Lax representation permits to pass from the original evolution defined by the equation to the evolution of some spectral data related to the problem $L \psi=0$ which is linear and consequently easily found. From this data the potentials can be recovered by a process called Inverse Scattering Method, see the monographs [5, 8].

The Generalized Zakharov-Shabat (GZS) system presented below is a paradigm of auxiliary linear problem. It can be written as follows

$$
L \psi=\left(\mathrm{i} \partial_{x}+q(x)-\lambda J\right) \psi=0 .
$$

Here $q(x)$ and $J$ belong to some fixed simple Lie algebra $\mathfrak{g}$ in some finite dimensional irreducible representation. The element $J$ is regular, that is the kernel of ad $J$ 
$\left(\operatorname{ad}_{J}(X) \equiv[J, X], X \in \mathfrak{g}\right)$ is the Cartan subalgebra $\mathfrak{h} \subset \mathfrak{g}$. The potential $q(x)$ belongs to the orthogonal completion $\mathfrak{h}^{\perp}$ of $\mathfrak{h}$ with respect to the Killing form

$$
\langle X, Y\rangle=\operatorname{tr}\left(\operatorname{ad}_{X} \operatorname{ad}_{Y}\right), \quad X, Y \in \mathfrak{g} .
$$

Therefore $q(x)=\sum_{\alpha \in \Delta} q_{\alpha} E_{\alpha}$ where $E_{\alpha}$ are the root vectors and $\Delta$ is the root system of $\mathfrak{g}$. The scalar functions $q_{\alpha}(x)$ defined on $\mathbb{R}$, are complex valued, smooth and rapidly vanishing for $x \rightarrow \pm \infty$ and we can assume that $q_{\alpha}(x)$ are of Schwartz type. The functions $q_{\alpha}$ are called also 'potentials' and we shall consider $q(x)$ as a point in an infinite dimensional manifold - the manifold of potentials. The classical Zakharov-Shabat system is obtained for $\mathfrak{g}=\mathfrak{s l}(2, \mathbb{C}), J=\operatorname{diag}(1,-1)$.

Remark 1. We assume that the basic properties of the semisimple Lie algebras (real and complex) are known. All definitions and normalizations we use coincide with those made in [13] and are almost universally accepted.

Remark 2. When Generalized Zakharov-Shabat systems on different algebras are involved we say that we have Generalized Zakharov-Shabat $\mathfrak{g}$-system to underline that it is on the algebra $\mathfrak{g}$, but when we work on a fixed algebra its symbol is usually omitted.

Referring for the details to [12] we simply remind that the adjoint solutions of GZS operator $L$ are functions of the type $w=m X m^{-1}$ where $X$ is a constant element from $\mathfrak{g}$ and $m$ is a fundamental solution of $L m=0$. Let us denote by $w^{\mathrm{a}}$ and $w^{\mathrm{d}}$ the orthogonal projection (with respect to the Killing form) of $w$ over $\mathfrak{h}^{\perp}$ and $\mathfrak{h}$ respectively. If one denotes the orthogonal projector on $\mathfrak{h}^{\perp}$ by $\pi_{0}$ then of course $w^{\mathrm{a}}=\pi_{0} w$ and $w^{\mathrm{d}}=\left(\mathbf{1}-\pi_{0}\right) w$. One of the most important fact from the theory of GZS system is that if a suitable set of adjoint solutions $\left(w_{i}(x, \lambda)\right)$ is taken then roughly speaking for $\lambda$ belonging to the spectrum of $L$ the functions $w_{i}^{\mathrm{a}}(x, \lambda)$ form a complete sets in the space of potentials. If one expands a potential over the subset of the adjoint solutions as coefficients one gets the minimal scattering data for $L$. Thus passing from the potentials to the scattering data can be considered as a sort of Fourier transform, called a generalized Fourier transform. For this transform the functions $w_{i}^{\mathrm{a}}(x, \lambda)$ play the role the exponents play in the usual Fourier transform. This interpretation was given for the first time in [1] and after that has been developed in a number of works (see for example the monographs $[8,15]$ for comprehensive study of $\mathfrak{s l}(2, \mathbb{C})$-case and bibliography and $[2,12]$ for more general situations).

I. The recursion operators (generating operators, $\Lambda$-operators) are the operators for which the functions $w_{i}^{\mathrm{a}}(x, \lambda)$ are eigenfunctions and therefore for the generalized Fourier transform they play the same role as the differentiation operator plays in 
the usual Fourier transform method. Their explicit form can be found in a number of articles, and books (see for example [8]). For the above reasons the recursion operators $\Lambda_{ \pm}$(usually one says just recursion operator) play an important role in the theory of soliton equations - it is a theoretical tool which apart from explicit solutions can give most of the information about the NLEEs, $[8,26]$. In particular, through them can be obtained

i) the hierarchies of the nonlinear evolution equations solvable through $L$

ii) the conservation laws for these NLEEs

iii) the hierarchies of Hamiltonian structures for these NLEEs.

There is another important trend in the theory of the recursion operators and it is related with the study of the recursion operators related to gauge-equivalent systems. Taking as example the GZS system, assume that we make a gauge transformation of the type $\psi \mapsto \psi_{0}^{-1} \psi=\tilde{\psi}$ where $\psi_{0}$ is a fundamental solution to GZS system corresponding to $\lambda=0$. Then if we denote $S=\psi_{0}^{-1} J \psi_{o}$ and the orbit of the coadjoint representation of the Lie group $G$ corresponding to $\mathfrak{g}$ by $\mathcal{O}_{J}$ we shall obtain that $\tilde{\psi}$ is a solution of the following linear problem

$$
\tilde{L} \tilde{\psi}=\mathrm{i} \partial_{x} \tilde{\psi}-\lambda S \tilde{\psi}=0, \quad S \in \mathcal{O}_{J}
$$

One can choose different fundamental solutions and one will obtain different behavior for $S$ when $x \mapsto \pm \infty$ but usually $\psi_{0}$ is taken to be the Jost solution that satisfies $\lim _{x \rightarrow-\infty} \psi_{0}=\mathbf{1}$. The system (3) is called GZS system in pole gauge in contrast to the system (1) which is called GZS system in canonical gauge.

The theory of the NLEEs related with the GZS auxiliary problem in canonical gauge $(L)$ is in direct connection with the theory of the NLEEs related with the GZS auxiliary problem in pole gauge $(\tilde{L})$. The NLEEs for both systems are in one-to-one correspondence and are called gauge-equivalent equations. This beautiful construction has been discovered for the first time in the famous work of $\mathrm{Za}$ kharov and Takhtadjan [25] in which there has been proved the gauge-equivalence of two famous equations - the Heisenberg ferromagnet equation and the nonlinear Schrödinger equation.

In fact the constructions for the system $L$ and its gauge equivalent $\tilde{L}$ are in complete analogy. Instead of the fixed Cartan subalgebra $\mathfrak{h}=\operatorname{kerad}_{J}$ we have 'moving' Cartan subalgebra $\mathfrak{h}_{S}(x)=\operatorname{kerad}_{S(x)}$, 'moving' space $\mathfrak{h}_{S}^{\perp}(x)$ orthogonal (with respect to the Killing form) to $\mathfrak{h}_{S}(x)$ (and consequently moving projector $\pi_{S}(x)$ ) etc. We have the corresponding adjoint solutions $\tilde{w}=\tilde{\psi} X \tilde{\psi}^{-1}$ where $\tilde{\psi}$ is a solution of $\tilde{L} \tilde{\psi}=0$ and $X$ is a constant element in $\mathfrak{g}$. If we denote by $\tilde{w}^{\mathrm{a}}$ and $\tilde{m}^{\mathrm{d}}$ 
the projections of $\tilde{w}(x)$ on $\mathfrak{h}_{S}^{\frac{1}{S}}(x)$ and $\mathfrak{h}_{S}(x)$ respectively then the corresponding recursion operators are constructed using the fact that the functions $\tilde{w}^{\mathrm{a}}$ must be eigenfunctions for them.

Let us make the following agreement. Though the Cartan subalgebra $\mathfrak{h}_{S}(x)$, its orthogonal space $\mathfrak{h}_{S}^{\frac{1}{S}}(x)$ and the projector $\pi_{S}(x)$, depend on $x$ we shall not write it explicitly unless there is a posibility of confusion. So for example in the case of a function $X(x)$ that is defined on $\mathbb{R}$ and such that $X(x) \in \mathfrak{h} \frac{\perp}{S}(x)$ we shall write simply $X \in \mathfrak{h} \frac{\perp}{S}$, for two functions $X(x)$ and $Y(x)$ we shall write instead of $X(x)=Y(x)$ simply $X=Y$ and so on.

For GZS system in pole gauge everything is easily reformulated and the only real difficulty is to calculate all the quantities that are expressed through $q$ and its derivative through $S$ and its derivatives. There is a clear procedure how to achieve that goal but in each particular case it requires new calculations. The procedure has been developed in detail in our $\mathrm{PhD}$ thesis [23], outlined in [9,10] (for the $\mathfrak{s l}(2, \mathbb{C})$ case) and in more general cases in [11]. In the case of $\mathfrak{s l}(3)$ the procedure has been carried out in detail in [24] - for all these references see also [8].

II. The recursion operators for GZS have also beautiful geometric meaning. It can be shown that their adjoint operators can be interpreted as Nijenhuis tensors on the manifolds of 'potentials' where the evolution defined by $[L, A]=0$ occurs. The point is that one of characteristic properties of the soliton equations is that they are not simply Hamiltonian but they are Hamiltonian with respect to two different compatible Poisson structures. This property is known as bi-Hamiltonian property of the NLEEs solvable through the corresponding linear problem. A Poisson structure on a manifold $\mathcal{M}$ is a field of linear maps $m \mapsto P_{m}: T_{m}^{*}(\mathcal{M}) \mapsto T_{m}(\mathcal{M})$ such that for any two smooth functions $f, g$ the expression $\{f, g\}(m)=\left\langle\mathrm{d} g_{m}, P_{m}(\mathrm{~d} f)_{m}\right\rangle$ is a Poisson bracket. (Here $\langle$,$\rangle is the canonical pairing between T_{m}(\mathcal{M})$ and $T_{m}^{*}(\mathcal{M})$ - the tangent and cotangent spaces at $m \in \mathcal{M})$. Compatible Poisson structures are called such Poisson structures $P, Q$ for which their linear combination $a P+b Q$ (where $a, b$ are constants) is also a Poisson tensor. It turns out that compatible Poisson structures give rise to Nijenhuis tensors in case one of it is invertible.

Indeed, if $Q$ is invertible, then one can define $N=P \circ Q^{-1}$ and $N$ is a field of linear maps $m \mapsto N_{m}: T_{m}(\mathcal{M}) \mapsto T_{m}(\mathcal{M})$ such that the so called Nijenhuis bracket $[N, N]$ of $N$ is zero. Then the manifold of potentials is endowed with a very special geometric structure - Poisson-Nijenhuis (P-N) structure of coupled Poisson tensor and a Nijenhuis tensor. The properties of the P-N structure are responsible for the fact that the symmetries of the soliton equations have 'hereditary' properties and that there are infinitely many Hamiltonian structures for the corresponding NLEEs. This interpretation was found by F. Magri in his pioneer works [16, 17], one can 
see all details of the theory in [4] or in [8] and we shall assume that it is known and shall not describe it again here.

As a matter of fact there is a nice picture of the relation of the P-N structures on the manifold of potentials for the GZS system in the canonical gauge, the manifold of potentials of the same system in pole gauge and the manifold of the corresponding Jost solutions, see [6, Ch. 15].

Together with the possibility to calculate the recursion operators for GZS system in pole gauge through the gauge transformation, there exists another option - to calculate directly the P-N structure on the manifold of potentials using the compatible Poisson structures and then to find the conjugate to the Nijenhuis tensor. In this work we shall use it and then shall compare our result with the Recursion Operator already known in the case $\mathfrak{s l}(3, \mathbb{C})$, see [24]. Our motivation comes from the fact that there has been some renewed interest in the GZS system in pole gauge and its reductions recently, see [6,7]. In the second of these works have been calculated the Recursion Operators in the case where some reductions are imposed on the $\mathfrak{s l}(3, \mathbb{C})$-GZS system in pole gauge using the usual technique and a technique developed in [14]. We intend to address this issue elsewhere, as here we shall concentrate on the $\mathfrak{s l}(3, \mathbb{C})$-GZS system in pole gauge in general position.

\section{P-N Structure for GZS Pole Gauge Hierarchy. The $\mathfrak{s}(3, \mathbb{C})$ Case}

Consider the GZS pole gauge $\mathfrak{s l}(3, \mathbb{C})$-system in general position - that is the smooth function $S(x)$ with domain $\mathbb{R}$, see (3), is subject only to the requirements that $S(x) \in \mathcal{O}_{J}$ and $S(x)$ tends fast enough to some constant values when $x \mapsto \pm \infty$. For $J$ we shall assume that $J=\operatorname{diag}\left(\lambda_{1}, \lambda_{2}, \lambda_{3}\right), \sum_{i} \lambda_{i}=0$, where all $\lambda_{i} \neq 0$. Of course $J$ must be regular, so that $\operatorname{kerad}_{J}$ coincides with the Cartan subalgebra of the diagonal matrices in $\mathfrak{s l}(3, \mathbb{C})$.

Let us consider a more general case then in the above when the algebra $\mathfrak{g}$ is arbitrary simple algebra. Let $S(x)$ is smooth, have values in $\mathfrak{g}$ and when $x \rightarrow \pm \infty$ the function $S(x)$ tends fast enough to constant values. Functions of this type form an infinite dimensional manifold which we shall denote by $\mathcal{M}$. Then it is reasonable to assume that the tangent space $T_{S}(\mathcal{M})$ at $S$ consists of all the smooth functions $X: \mathbb{R} \mapsto \mathfrak{g}$ vanishing fast enough when $x \mapsto \pm \infty$. We denote that space by $\mathfrak{F}(\mathfrak{g})$. We shall also assume that the 'dual space' $T_{S}^{*}(\mathcal{M})$ is equal to $\mathfrak{F}(\mathfrak{g})$ and if $\alpha \in T_{S}^{*}(\mathcal{M}), X \in T_{S}(\mathcal{M})$ then

$$
\alpha(X)=\langle\langle\alpha, X\rangle\rangle \equiv \int_{-\infty}^{+\infty}\langle\alpha(x), X(x)\rangle \mathrm{d} x
$$


where $\langle$,$\rangle is the Killing form of \mathfrak{s l}(3, \mathbb{C})$.

Remark 3. We identify $T_{S}^{*}(\mathcal{M})$ and $T_{S}(\mathcal{M})$ using the bi-linear form $\langle\langle\rangle$,$\rangle . We do$ not want to make the definitions more precise, since we will speak rather about a geometric picture then about precise results. Such results can be obtained only after profound study of the spectral theory of $L$ and $\tilde{L}$. In particular, we put dual space in quotation marks because it is clearly not equal to the dual of $\mathfrak{F}(\mathfrak{g})$. We mention however that the term 'allowed' functional $H$ means that $\frac{\delta H}{\delta S} \in$ $T_{S}^{*}(\mathcal{M}) \sim T_{S}(\mathcal{M})$.

First we note that the operators

$$
\begin{gathered}
\alpha \mapsto P(X)=\mathrm{i}_{x} \alpha, \quad \alpha \in T_{S}^{*}(\mathcal{M}) \\
\alpha \mapsto Q(\alpha)=\operatorname{ad}_{S}(\alpha), \quad S \in \mathcal{M} .
\end{gathered}
$$

It is a fact from the general theory that these Poisson tensors are compatible, see [6, Ch. 15]. In other words $P+Q$ is also a Poisson tensor. Let us also mention that the tensor $Q$ is the canonical Kirillov tensor which acquires the above form because the algebra is simple and the coadjoint and the adjoint representation are equivalent.

Now let $\mathcal{O}_{J}$ be the orbit of the coadjoint representation of $G$ (the group that corresponds to $\mathfrak{g}$ ) passing through $J$. Let us consider the set of smooth functions $f: \mathbb{R} \mapsto \mathcal{O}_{J}$ such that when $x \rightarrow \pm \infty$ they tend fast enough to constant values. The set of this functions is denoted by $\mathcal{N}$ and clearly can be considered as submanifold of $\mathcal{M}$. If $S \in \mathcal{N}$ the tangent space $T_{S}(\mathcal{N})$ consists of all smooth functions $X$, tending to zero fast enough when $x \mapsto \pm \infty$ and such that $X(x) \in T_{S(x)}\left(\mathcal{O}_{J}\right)$ (Recall that $\mathcal{O}_{J}$ is a smooth manifold in the classical sense.) We assume again that $T_{S}^{*}(\mathcal{N}) \sim T_{S}(\mathcal{N})$ and that these spaces are identified via $\langle\langle\rangle$,$\rangle .$

The Poisson tensors $P$ and $Q$ can be restricted from $\mathcal{M}$ to $\mathcal{N}$. The question how to restrict a Poisson tensor on submanifold has been considered in detail in the literature, see for example [20-22]. We shall use a simplified version of the results obtained in these papers, proved in $[18,19]$. We call it first restriction theorem.

Theorem 4. Let $\mathcal{M}$ be Poisson manifold with Poisson tensor $P$ and $\overline{\mathcal{M}} \subset \mathcal{M}$ be a submanifold. Let us denote by $j$ the inclusion map of $\overline{\mathcal{M}}$ into $\mathcal{M}$, by $\mathcal{X}_{P}^{*}(\overline{\mathcal{M}})_{m}$ the subspace of covectors $\alpha \in T_{m}^{*}(\mathcal{M})$ such that

$$
P_{m}(\alpha) \in \mathrm{d} j_{m}\left(T_{m}(\overline{\mathcal{M}})\right)=\operatorname{Im}\left(\mathrm{d} j_{m}\right), \quad m \in \overline{\mathcal{M}}
$$

Here $\operatorname{Im}$ denotes the image and $T^{\perp}(\overline{\mathcal{M}})_{m}$ - the set of all covectors at $m \in \mathcal{M}$ vanishing on the subspace $\operatorname{Im}\left(\mathrm{d} j_{m}\right), m \in \overline{\mathcal{M}}$ is called also the annihilator of 
$\operatorname{Im}\left(\mathrm{d} j_{m}\right)$ in $T_{m}^{*}(\mathcal{M})$. Let the following relations hold

$$
\begin{gathered}
\mathcal{X}_{P}^{*}(\overline{\mathcal{M}})_{m}+T^{\perp}(\overline{\mathcal{M}})_{m}=T_{m}^{*}(\mathcal{M}), \quad m \in \overline{\mathcal{M}} \\
\mathcal{X}_{P}^{*}(\overline{\mathcal{M}})_{m} \cap T^{\perp}(\overline{\mathcal{M}})_{m} \subset \operatorname{ker}\left(P_{m}\right)
\end{gathered}
$$

Then there exists unique Poisson tensor $\bar{P}$ on $\overline{\mathcal{M}}, j$-related with $P$, that is

$$
P_{m}=\mathrm{d} j_{m} \circ \bar{P}_{m} \circ\left(\mathrm{d} j_{m}\right)^{*} .
$$

The proof of the theorem is constructive. First, one takes $\beta \in T_{m}^{*}(\overline{\mathcal{M}})$, then represents $\left(j^{*} \beta\right)_{m}$ as $\alpha_{1}+\alpha_{2}$ where $\alpha_{1} \in \mathcal{X}_{P}^{*}(\overline{\mathcal{M}})_{m}, \alpha_{2} \in T^{\perp}(\overline{\mathcal{M}})_{m}$ and finally puts $\bar{P}_{m}(\beta)=P_{m}\left(\alpha_{1}\right)$ (we identify $m$ and $j(m)$ here).

Restricting the Poisson tensor $Q$ is easy, one readily get that the restriction $\bar{Q}$ is given by the same formula as before

$$
\alpha \mapsto \bar{Q}(\alpha)=\operatorname{ad}_{S}(\alpha), \quad S \in \mathcal{N}, \quad \alpha \in T_{S}^{*}(\mathcal{N}) .
$$

The tensor $P$ is a little harder to restrict. The restriction we present below has been preformed in various works in the simplest case $\mathfrak{g}=\mathfrak{s l}(2, \mathbb{C})$, see for example [19]. We do it now in the case $\mathfrak{g}=\mathfrak{s l}(3, \mathbb{C})$, in other words starting from here the Lie algebra $\mathfrak{g}$ will be $\mathfrak{s l}(3, \mathbb{C})$.

First, let us introduce some facts and notation. Since $J$ is a regular element from the Cartan subalgebra $\mathfrak{h}$ then each element $S$ from the orbit $\mathcal{O}_{J}$ is also regular, $\mathfrak{h}_{S}(x)=\operatorname{kerad}_{S(x)}$ is a Cartan subalgebra of $\mathfrak{s l}(3, \mathbb{C})$ and we have

$$
\mathfrak{s l}(3, \mathbb{C})=\mathfrak{h}_{S}(x) \oplus \mathfrak{h}_{S}^{\perp}(x) .
$$

If $X \in T_{S}(\mathcal{N})=\mathfrak{h}_{S}^{\perp}$ then $X(x) \in \mathfrak{h} \frac{1}{S}(x)$ (we recall that these spaces depend on the point $x$ ) but in addition $X$ is smooth and vanishes rapidly when $x \mapsto \pm \infty$. We shall denote the set of these functions by $\mathfrak{F}\left(\mathfrak{h} \frac{\perp}{S}\right)$. So according to our notation $X \in \mathfrak{h} \frac{\perp}{S}$ and $X \in \mathfrak{F}\left(\mathfrak{h} \frac{\perp}{S}\right)$. Using the same logic, for $X \in \mathfrak{F}\left(\mathfrak{h} \frac{\perp}{S}\right)$ we write $\operatorname{ad}_{S}(X)$ which means the function $\operatorname{ad}_{S(x)} X(x)$ belonging to $\mathfrak{F}\left(\mathfrak{h} \frac{\perp}{S}\right)$.

We have some facts about $J$ that we introduce in the propositions below. For the proofs see [24].

Proposition 5. The matrices $J$ and $J_{1}=J^{2}-\frac{1}{3} \operatorname{tr}\left(J^{2}\right) \mathbf{1}$ span the Cartan subalgebra $\mathfrak{h}=\operatorname{kerad}_{J}$.

As a consequence, for $S \in \mathcal{O}_{J}$ the matrices $S$ and $S_{1}=S^{2}-\frac{2}{3} \mathbf{1}$ span the Cartan subalgebra $\mathfrak{h}_{S}$ of $\mathfrak{s l}(3, \mathbb{C})$. On $\mathfrak{h} \frac{1}{S}$ the operator $\operatorname{ad}_{S}$ is invertible. 
Proposition 6. The matrix $J$ satisfies the equation

$$
J^{3}=\frac{1}{2} C_{2} J+\frac{1}{3} C_{3} \mathbf{1}, \quad C_{2}=\lambda_{1}^{2}+\lambda_{2}^{2}+\lambda_{3}^{2}, \quad C_{3}=\lambda_{1}^{3}+\lambda_{2}^{3}+\lambda_{3}^{3} .
$$

Proposition 7. If $S \in \mathcal{O}_{J}=\left\{\tilde{X} ; \tilde{X}=g J g^{-1}, g \in \mathrm{SL}(3, \mathbb{C})\right\}$ then $S$ satisfies (13), that is $S^{3}=\frac{1}{2} C_{2} S+\frac{1}{3} C_{3} 1$. If in addition for all $\lambda_{i}, \lambda_{i} \neq 0$ the inverse is also true, that is any $S$ that satisfies the equation $S^{3}=\frac{1}{2} C_{2} S+\frac{1}{3} C_{3} 1$ belongs to the orbit.

The Killing form of $\mathfrak{s l}(3, \mathbb{C})$ is equal to $6 \operatorname{tr} X Y$ and one has the following useful identities

$$
\langle J, J\rangle=6 C_{2}, \quad\left\langle J_{1}, J_{1}\right\rangle=C_{2}^{2}, \quad\left\langle J, J_{1}\right\rangle=6 C_{3} .
$$

The Killing form is invariant with respect to the adjoint action, so we also have

$$
\langle S, S\rangle=6 C_{2}, \quad\left\langle S_{1}, S_{1}\right\rangle=C_{2}^{2}, \quad\left\langle S, S_{1}\right\rangle=6 C_{3} .
$$

The Gram matrix

$$
T=\left(\begin{array}{cc}
\langle J, J\rangle & \left\langle J, J_{1}\right\rangle \\
\left\langle J_{1}, J\right\rangle & \left\langle J_{1}, J_{1}\right\rangle
\end{array}\right)=\left(\begin{array}{cc}
6 C_{2} & 6 C_{3} \\
6 C_{3} & C_{2}^{2}
\end{array}\right)
$$

has determinant $d_{1}=6\left(C_{2}^{3}-6 C_{3}^{2}\right)$. Of course $d_{1} \neq 0$. One can show that

$$
d_{1}=12\left(\lambda_{1}-\lambda_{2}\right)^{2}\left(\lambda_{2}-\lambda_{3}\right)^{2}\left(\lambda_{1}-\lambda_{3}\right)^{2} \equiv 12 d .
$$

Therefore

$$
T^{-1}=\frac{1}{12 d}\left(\begin{array}{cc}
\left\langle J_{1}, J_{1}\right\rangle & -\left\langle J, J_{1}\right\rangle \\
-\left\langle J_{1}, J\right\rangle & \langle J, J\rangle
\end{array}\right)=\frac{1}{12 d}\left(\begin{array}{cc}
C_{2}^{2} & -6 C_{3} \\
-6 C_{3} & 6 C_{2}
\end{array}\right) .
$$

Now we are in position to perform the restriction of $P$ on $\mathcal{N}$. For $S \in \mathcal{N}$ we have

$$
\begin{aligned}
\mathcal{X}_{P}^{*}(\mathcal{N})_{S} & =\left\{\alpha ; \mathrm{i} \partial_{x} \alpha \in \mathfrak{F}\left(\mathfrak{h}^{\perp}\right)\right\} \\
T^{\perp}(\mathcal{N})_{S} & =\left\{\alpha ;\langle\langle\alpha, X\rangle\rangle=0, X \in \mathfrak{F}\left(\mathfrak{h}_{S}^{\perp}\right)\right\} .
\end{aligned}
$$

We see that $T^{\perp}(\mathcal{N})_{S}$ is the set of smooth functions $\alpha(x)$ such that $\alpha \in \mathfrak{h}_{S}$ tends to zero fast enough when $x \mapsto \pm \infty$. We shall denote this space by $\mathfrak{F}\left(\mathfrak{h}_{S}\right)$. Naturally, $\mathfrak{F}\left(\mathfrak{h}_{S}\right) \subset \mathfrak{F}\left(\mathfrak{h}_{S}\right)_{0}$, where the space $\mathfrak{F}\left(\mathfrak{h}_{S}\right)_{0}$ consists of all smooth functions $X(x)$ such that $X \in \mathfrak{h}$ and such that $X$ tends to some constant values when $x \mapsto \pm \infty$. Since $S$ and $S_{1}$ span $\mathfrak{h}_{S}$, we have that $S, S_{1} \in \mathfrak{F}\left(\mathfrak{h}_{S}\right)_{0}$ and

$$
\begin{aligned}
\mathfrak{F}\left(\mathfrak{h}_{S}\right)_{0}= & \left\{X ; X=a(x) S(x)+b(x) S_{1}(x), \quad a(x), b(x)-\right.\text { smooth } \\
& a(x), b(x) \text { tend to some constant values when } x \mapsto \pm \infty\} \\
\mathfrak{F}\left(\mathfrak{h}_{S}\right)= & \left\{X ; X=a(x) S(x)+b(x) S_{1}(x), \quad a(x), b(x)-\right.\text { smooth } \\
& \left.\lim _{x \rightarrow \pm \infty} a(x)=\lim _{x \rightarrow \pm \infty} b(x)=0\right\} .
\end{aligned}
$$


Let us consider now $\mathcal{X}_{P}^{*}(\mathcal{N})_{S} \cap T^{\perp}(\mathcal{N})_{S}$. It consists of the elements

$$
\alpha=a(x) S(x)+b(x) S_{1}(x)
$$

such that $\mathrm{i} \partial_{x} \alpha \in \mathfrak{F}\left(\mathfrak{h} \frac{\perp}{S}\right)$. But

$$
\mathrm{i} \partial_{x} \alpha=\mathrm{i} a(x) S_{x}+\mathrm{i} b(x)\left(S_{1}\right)_{x}+\mathrm{i} a_{x} S(x)+\mathrm{i} b_{x} S_{1}(x)
$$

so we must have $\left\langle\mathrm{i} \partial_{x} \alpha(x), S(x)\right\rangle=\left\langle\mathrm{i} \partial_{x} \alpha(x), S_{1}(x)\right\rangle=0$. Now, let us note that from (15) follows that

$$
\left\langle S(x), S_{x}(x)\right\rangle=\left\langle S_{1}(x),\left(S_{1}\right)_{x}(x)\right\rangle=0,\left\langle S_{1}(x), S_{x}(x)\right\rangle=-\left\langle\left(S_{1}\right)_{x}(x), S_{x}(x)\right\rangle .
$$

Next

$$
\left\langle S_{1}, S_{x}\right\rangle=6 \operatorname{tr}\left(S_{x} S^{2}\right)=2 \operatorname{tr}\left(S^{3}\right)_{x} .
$$

Using Proposition 7 we get that $\left\langle S_{1}, S_{x}\right\rangle$ is proportional to $\operatorname{tr} S_{x}=0$. In this way we see that $S_{x},\left(S_{1}\right)_{x}$ belong to $\mathfrak{F}\left(\mathfrak{h}_{S}^{\perp}\right)$ and therefore $a_{x}=b_{x}=0$. Then $a$ and $b$ can be only identically zero and

$$
\mathcal{X}_{P}^{*}(\mathcal{N})_{S} \cap T^{\perp}(\mathcal{N})_{S}=\{0\} \subset \operatorname{ker} P_{S}
$$

Consider now arbitrary $\alpha \in T^{*}(\mathcal{N})_{S}$. We want to represent it as $\alpha_{1}+\alpha_{2}$, where $\alpha_{1} \in \mathcal{X}^{*}(\mathcal{N})_{S}, \alpha_{2} \in T^{\perp}(\mathcal{N})_{S}$. Therefore, $\alpha_{2}=A(x) S(x)+B(x) S_{1}$ with $A(x), B(x)$ vanishing when $x \mapsto \pm \infty$. In addition, we must have

$$
\mathrm{i} \partial_{x} \alpha=\mathrm{i} \partial_{x} \alpha_{1}+\mathrm{i} A(x) S_{x}+\mathrm{i} B(x)\left(S_{1}\right)_{x}+\mathrm{i} A_{x} S(x)+\mathrm{i} B_{x} S_{1}
$$

where $\mathrm{i} \partial_{x} \alpha_{1} \in \mathfrak{F}\left(\mathfrak{h} \frac{\perp}{S}\right)$. Taking the Killing form with $S$ and $S_{1}$ we get the system

$$
\begin{aligned}
\left\langle\partial_{x} \alpha, S(x)\right\rangle & =A_{x}\langle J, J\rangle+B_{x}\left\langle J, J_{1}\right\rangle \\
\left\langle\partial_{x} \alpha, S_{1}(x)\right\rangle & =A_{x}\left\langle J, J_{1}\right\rangle+B_{x}\left\langle J_{1}, J_{1}\right\rangle
\end{aligned}
$$

and therefore

$$
\left(\begin{array}{c}
A_{x} \\
B_{x}
\end{array}\right)=T^{-1}\left(\begin{array}{c}
\left\langle\partial_{x} \alpha, S(x)\right\rangle \\
\left\langle\partial_{x} \alpha, S_{1}(x)\right\rangle
\end{array}\right)
$$

where $T$ is the Gram matrix introduced earlier. So we obtain

$$
\left(\begin{array}{c}
A \\
B
\end{array}\right)=T^{-1}\left(\begin{array}{c}
\partial_{x}^{-1}\left\langle\partial_{x} \alpha, S(x)\right\rangle \\
\partial_{x}^{-1}\left\langle\partial_{x} \alpha, S_{1}(x)\right\rangle
\end{array}\right) .
$$


Remark 8. Usually in all theory of the recursion operators and their geometric interpretation the expressions on which the operator $\partial_{x}^{-1}$ acts are total derivatives. Thus the same results will be obtained choosing for $\partial_{x}^{-1}$ any of the following operators

$$
\int_{-\infty}^{x} \cdot \mathrm{d} y, \quad \int_{+\infty}^{x} \cdot \mathrm{d} y, \quad \frac{1}{2}\left(\int_{-\infty}^{x} \cdot \mathrm{d} y+\int_{+\infty}^{x} \cdot \mathrm{d} y\right)
$$

However, one uses more frequently the third expression when one writes the corresponding Poisson tensors in order to make them explicitly skew-symmetric.

Returning to our task, for $\alpha \in T^{*}(\mathcal{N})_{S}$ let us put

$$
\begin{aligned}
& \alpha_{1}=\alpha-\alpha_{2} \\
& \alpha_{2}=\left(S, S_{1}\right) T^{-1}\left(\begin{array}{c}
\partial_{x}^{-1}\left\langle\partial_{x} \alpha, S(x)\right\rangle \\
\partial_{x}^{-1}\left\langle\partial_{x} \alpha, S_{1}(x)\right\rangle
\end{array}\right) .
\end{aligned}
$$

One checks that $\alpha_{1}, \alpha_{2}$ lie in the spaces $\mathcal{X}^{*}(\mathcal{N})_{S}, T^{\perp}(\mathcal{N})_{S}$ respectively. Thus the conditions of the first restriction theorem are fulfilled. Noting that for $\beta \in T_{S}^{*}(\mathcal{N})$ we have $\mathrm{d} j_{S}^{*} \beta=\pi_{S}(\beta)$ we find that the restriction $\bar{P}$ of $P$ on $\mathcal{N}$ has the form

$$
\bar{P}(\beta)=\mathrm{i} \pi_{S} \partial_{x} \beta-\mathrm{i}\left(S_{x},\left(S_{1}\right)_{x}\right) T^{-1}\left(\begin{array}{c}
\partial_{x}^{-1}\left\langle\partial_{x} \beta, S(x)\right\rangle \\
\partial_{x}^{-1}\left\langle\partial_{x} \beta, S_{1}(x)\right\rangle
\end{array}\right) .
$$

The Poisson tensor $\bar{Q}$ is invertible on $\mathcal{N}$, so one can construct a Nijenhuis $N=$ $\bar{P} \circ \operatorname{ad}_{S}^{-1}$ tensor which evaluated at $X \in \mathfrak{F}\left(\mathfrak{h} \frac{\perp}{S}\right)$ gives

$$
N(X)=\mathrm{i} \pi_{S} \partial_{x}\left(\operatorname{ad}_{S}^{-1} X\right)-\mathrm{i}\left(S_{x},\left(S_{1}\right)_{x}\right) T^{-1}\left(\begin{array}{c}
\partial_{x}^{-1}\left\langle\partial_{x}\left(\operatorname{ad}_{S}^{-1} X\right), S(x)\right\rangle \\
\partial_{x}^{-1}\left\langle\partial_{x}\left(\operatorname{ad}_{S}^{-1} X\right), S_{1}(x)\right\rangle
\end{array}\right)
$$

Taking into account that $\left\langle\operatorname{ad}_{S}^{-1}(X), S\right\rangle=\left\langle\operatorname{ad}_{S}^{-1}(X), S_{1}\right\rangle=0$ the above can be cast into the equivalent form

$$
N(X)=\mathrm{i} \pi_{S} \partial_{x}\left(\operatorname{ad}_{S}^{-1} X\right)+\mathrm{i}\left(S_{x},\left(S_{1}\right)_{x}\right) T^{-1}\left(\begin{array}{c}
\left.\partial_{x}^{-1}\left\langle\operatorname{ad}_{S}^{-1} X\right) S_{x}(x)\right\rangle \\
\partial_{x}^{-1}\left\langle\operatorname{ad}_{S}^{-1} X,\left(S_{1}\right)_{x}(x)\right\rangle
\end{array}\right) .
$$

From the general theory of the compatible Poisson tensors now follows

Theorem 9. The Poisson tensor field $\bar{Q}$ and the Nijenhuis tensor field $N$ endow the manifold $\mathcal{N}$ with a $\mathrm{P}-\mathrm{N}$ structure. 
The final step is to calculate the dual of the tensor $N$ with respect to the pairing $\langle\langle\rangle$,$\rangle . A quick calculation, taking into account that \operatorname{ad}_{S}$ is skew-symmetric with respect to the Killing form, gives for $\alpha \in \mathfrak{F}\left(\mathfrak{h} \frac{\perp}{S}\right)$

$$
N^{*}(\alpha)=\operatorname{iad}_{S}^{-1}\left[\pi_{S} \partial_{x} \alpha+\left(S_{x},\left(S_{1}\right)_{x}\right) T^{-1}\left(\begin{array}{c}
\partial_{x}^{-1}\left\langle\alpha S_{x}(x)\right\rangle \\
\partial_{x}^{-1}\left\langle\alpha,\left(S_{1}\right)_{x}(x)\right\rangle
\end{array}\right)\right]
$$

or equivalently

$$
N^{*}(\alpha)=\operatorname{iad}_{S}^{-1}\left[\pi_{S} \partial_{x} \alpha-\left(S_{x},\left(S_{1}\right)_{x}\right) T^{-1}\left(\begin{array}{c}
\partial_{x}^{-1} \partial_{x}\langle\alpha S(x)\rangle \\
\partial_{x}^{-1}\left\langle\partial_{x} \alpha, S_{1}(x)\right\rangle
\end{array}\right)\right] .
$$

But if we write the above in components we shall see that these are the recursion operators $\tilde{\Lambda}_{ \pm}$for the GZS system in pole gauge, see [24]. Thus our results confirm the idea that the recursion operators and the Nijenhuis tensors are dual objects.

The theory will not be complete if we are not able to present the hierarchies of integrable equations. Geometrically, they are the vector fields for the P-N structure, that is such that $L_{Z} \bar{Q}=0, L_{Z} N=0$ where $L_{Z}$ denotes the Lie derivative. Fortunately, from the general theory, see [8], we have that the hierarchies of the soliton equations are generated by the fields

$$
X_{k}^{a, b}=N^{k}\left[S, a J+b J^{2}\right], \quad a, b=\text { const }, \quad k=0,1,2, \ldots
$$

Thus the last thing that remains to be done is to express the operator $\operatorname{ad}_{S}^{-1}$ through $S$. For this let us note that if all the eigenvalues of $J$ are different then the operators $\operatorname{ad}_{J}$ and $\operatorname{ad}_{J}^{-1}\left(\operatorname{ad}_{S}\right.$ and $\operatorname{ad}_{S}^{-1}$ respectively) are semisimple and have common eigenvectors. One then can apply the spectral decomposition theorem for the operator $\operatorname{ad}_{S}$. The procedure is described in details in [24], and here we shall give the final result

$$
\operatorname{ad}_{S}^{-1}=l\left(\operatorname{ad}_{S}\right)
$$

where $l(\lambda)$ is the polynomial

$$
l(\lambda)=\frac{\lambda}{d}\left(\lambda^{2}-\frac{3}{2} C_{2}\right)^{2}
$$

and

$$
d=\left(\lambda_{1}-\lambda_{2}\right)^{2}\left(\lambda_{2}-\lambda_{3}\right)^{2}\left(\lambda_{1}-\lambda_{3}\right)^{2} .
$$

Let us make an important observation. As $l(\lambda)$ is of the form $\lambda l_{0}(\lambda)$, where $l_{0}$ is another polynomial, then we have $l\left(\operatorname{ad}_{S}\right)=l_{0}\left(\operatorname{ad}_{S}\right) \operatorname{ad}_{S}$. Therefore we have $l\left(\operatorname{ad}_{S}\right) \tilde{\pi}_{0}=l\left(\operatorname{ad}_{S}\right)$. The formula for $\operatorname{ad}_{S}^{-1}$ is true irrespective of the choice of the representation. However, we are working in a fixed representation and can 
simplify (37) using the fact that $S^{3}=\frac{1}{2} C_{2} S+\frac{1}{3} C_{3} 1$. Each time we have $S^{3}$ we can substitute it with $\frac{1}{2} C_{2} S+\frac{1}{3} C_{3} \mathbf{1}$ and get expressions whose degree in $S$ is at most 2 . Thus finally we obtain

$$
\operatorname{ad}_{S}^{-1} \tilde{X}=\frac{1}{d}\left\{C_{2}^{2}[S, \tilde{X}]+\frac{3}{2} C_{2} S[S, \tilde{X}] S-3 C_{3}\left[S^{2}, \tilde{X}\right]\right\}, \quad \tilde{X} \in \mathfrak{h} \frac{\perp}{S}
$$

In order to express everything in (36) through $S$ and its derivatives we need to calculate $\operatorname{ad}_{S}^{-1} S_{x}$ and $\operatorname{ad}_{S}^{-1}\left(S_{1}\right)_{x}$. We have

$$
\begin{aligned}
& \operatorname{ad}_{S}^{-1} S_{x}=-3 C_{3}\left[S^{2}, S_{x}\right]+C_{2}^{2}\left[S, S_{x}\right]+\frac{3}{2} C_{2} S\left[S, S_{x}\right] S \\
& \operatorname{ad}_{S}^{-1}\left(S_{1}\right)_{x}=C_{2}^{2}\left[S^{2}, S_{x}\right]-2 C_{2} C_{3}\left[S, S_{x}\right]-3 C_{3} S\left[S, S_{x}\right] S .
\end{aligned}
$$

Since $d=C_{2}^{2} / 2-3 C_{3}^{2}$ from this system we get two simple formulae

$$
\begin{aligned}
& \operatorname{ad}_{S}^{-1}\left[\frac{C_{2}}{2}\left(S^{2}\right)_{x}+C_{3} S_{x}\right]=\left[S^{2}, S_{x}\right] \\
& \operatorname{ad}_{S}^{-1}\left[\frac{C_{2}^{2}}{3} S_{x}+C_{3}\left(S^{2}\right)_{x}\right]=\frac{2 C_{2}}{3}\left[S, S_{x}\right]+S\left[S, S_{x}\right] S .
\end{aligned}
$$

These relations were obtained also in [24], but actually they were obtained earlier in a different way and in a different form in [3]. For example, in [3] in the right hand side of (43) instead of the expression $(2 / 3) C_{2}\left[S, S_{x}\right]+S\left[S, S_{x}\right] S$ stands

$$
\left[S,\left\{S,\left\{S, S_{x}\right\}\right\}\right]+\frac{1}{6} C_{2}\left[S, S_{x}\right]
$$

where $\{$,$\} means anticommutator. However, it is not difficult to check that because$ of the relation $S^{3}=(1 / 2) C_{2} S-(1 / 3) C_{3} \mathbf{1}$, these expressions are equal.

The equations from the two-parameter hierarchy (36) are rather complicate for calculation and are calculated up to the equations involving second order derivatives in $x$. Some simplification can be achieved using (42), see [24]. Two particular cases can be selected

$$
\begin{gathered}
\partial_{t} S=-\mathrm{i} a\left(\left[S^{2}, S_{x x}\right]+\left[S, S_{x}^{2}\right]\right), \quad a=\text { const } \\
S_{t}=-\mathrm{i} b\left(\frac{2}{3} C_{2}\left[S, S_{x x}\right]+S_{x}\left[S, S_{x}\right] S+S\left[S, S_{x x}\right] S\right), \quad b=\text { const. }
\end{gathered}
$$

If one prefers the equation (46) can be cast in the equivalent form

$$
S_{t}=-\mathrm{i} b\left(\frac{1}{6} C_{2}\left[S, S_{x x}\right]+\left[S,\left\{S,\left(S^{2}\right)_{x x}\right\}\right]\right) .
$$


According to [3], when i $S$ belongs to the compact real form $\mathfrak{s u}(3)$ of $\mathfrak{s l}(3, \mathbb{C})$ the equations (45) - (46) describe the dynamics of spin systems with spin 1 . Imposing i $S \in \mathfrak{s u}(3)$ does not affect any of the relations we have already. Indeed, if $J^{\dagger}=J$, $q^{\dagger}=q$ (that is, if $\left.\mathrm{i} J \in \mathfrak{s u}(3), \mathrm{i} q \in \mathfrak{s u}(3)\right)$ then $\psi_{0}$ belongs to the group $\mathrm{SU}(3)$ and therefore $\mathrm{i} S=\mathrm{i} \psi_{0}^{-1} J \psi_{0} \in \mathfrak{s u}(3)$. In other words the requirements $\mathrm{i} J \in \mathfrak{s u}(3)$, $\mathrm{i} q \in \mathfrak{s u}(3)$ impose algebraical restriction compatible with all our constructions.

\section{Conclusion}

In this article we have found the P-N structure on the manifold of potentials $\mathcal{N}$ for the GZS system in pole gauge on the Lie algebra $\mathfrak{s l}(3, \mathbb{C})$ obtaining geometric interpretation of the Recursion Operators and the corresponding hierarchies of soliton equations.

\section{Acknowledgment}

This work has been essentially supported by the incentive grant \# 73026 of NRF South Africa.

\section{References}

[1] Ablowitz M., Kaup D., Newell A. and Segur H., The Inverse Scattering Problem - Fourier Analysis for Nonlinear Problems, Studies in Appl. Math. 53 (1974) 249-315.

[2] Beals R. and Coifman R., Scattering and Inverse Scattering for First Order Systems, Comm. Pure \& Appl. Math. 37 (1984) 39-89.

[3] Borovik A. and Popkov V., Completely Integrable Spin - 1 Chains, Sov. Phys. JETPH 71 (1990) 177-185.

[4] Casati P., Falqui G., Magri F. and Pedroni M., Eight Lectures on Integrable Systems. Integrability of Nonlinear Systems, In: LNP vol. 495, Y. KosmannSchwarzbach, B. Grammaticos and K. Tamizhmani (Eds), Springer, Berlin 2004, pp 209-250.

[5] Faddeev L. and Takhtadjan L., Hamiltonian Method in the Theory of Solitons, Springer, Berlin 1987.

[6] Gerdjikov V., Mikhailov A. and Valchev T., Reductions of Integrable Equations on A.III - Symmetric Spaces, J. Phys. A: Math. \& Theor. 43 (2010) 434015. 
[7] Gerdjikov V., Mikhailov A. and Valchev T., Recursion Operators and Reductions of Integrable Equations on Symmetric Spaces, J. Geom. Symm. Phys. 20 (2010) 1-34.

[8] Gerdjikov V., Vilasi G. and Yanovski A., Integrable Hamiltonian Hierarchies - Spectral and Geometric Methods, Springer, Heidelberg 2008.

[9] Gerdjikov V. and Yanovski A., Gauge-Covariant Theory of the Generating Operator. I., Comm. Math. Phys. 103 (1986) 549-56.

[10] Gerdjikov V. and Yanovski A., Gauge-Covariant Formulation of the Generating Operator. 1. The Zakharov-Shabat System, Phys. Lett. A 103 (1984) 232-236.

[11] Gerdjikov V. and Yanovski A., Gauge-Covariant Formulation of the Generating Operator. 2. Systems on Homogeneous Spaces, Phys. Lett. A 110 (1985) 53-57.

[12] Gerdjikov V. and Yanovski A., Completeness of the Eigenfunctions for the Caudrey-Beals-Coifman System, J. Math. Phys. 35 (1994) 3687-3721.

[13] Goto M. and Grosshans F., Semisimple Lie Algebras, Lecture Notes in Pure and Applied Mathematics vol. 38, M. Dekker, New York 1978.

[14] Gürses M., Karasu A. and Sokolov V., On Construction of Recursion Operators from Lax Representation, J. Math. Phys. 40 (1999) 6473-6490.

[15] Iliev I., Khristov E. and Kirchev K., Spectral Methods in Soliton Equations, Pitman Monographs and Surveys in Pure and Applied Mathematics vol.73, John Wiley \& Sons, New-York 1994.

[16] Magri F., A Simple Model of the Integrable Hamiltonian Equations, J. Math. Phys. 19 (1978) 1156-1162.

[17] Magri F., A Geometrical Approach to the Nonlinear Solvable Equations, In: Lecture Notes in Physics vol.120, Springer, Berlin 1980.

[18] Magri F. and Morosi C., A Geometrical Characterization of Integrable Hamiltonian Systems Through the Theory of Poisson-Nijenhuis Manifolds, Quaderni del Dipartimento di Matematica, Università di Milano 1984.

[19] Magri F., Morosi C. and Ragnisco O., Reduction Techniques for InfiniteDimensional Hamiltonian Systems: Some Ideas and Applications, Comm. Math. Phys. 99 (1985) 115-140.

[20] Marsden J. and Ratiu T., Reduction of Poisson Manifolds, Lett. Math. Phys. 11 (1986) 161-169.

[21] Ortega J. and Ratiu T., Momentum Maps and Hamiltonian Reduction, Progress in Mathematics vol. 222, Birkhäuser, Boston 2004. 
[22] Ortega J. and Ratiu T., Singular Reduction of Poisson Manifolds, Lett. Math. Phys. 46 (1998) 59-372.

[23] Yanovski A., Gauge-Covariant Approach to the Theory of the Generating Operators for Soliton Equations, PhD thesis: 5-87-222, Joint Institute for Nuclear Research (JINR), 1987.

[24] Yanovski A., Generating Operators for the Generalized Zakharov-Shabat System and its Gauge Equivalent System in $\mathfrak{s l}(3, \mathbb{C})$ Case, Universität Leipzig, Naturwissenchaftlich Theoretisches Zentrum Report \# 20, 1993, http://cdsweb.cern.ch/record/256804/files/P00019754.pdf.

[25] Zakharov V. and Takhtadjan L., Equivalence Between Nonlinear Schrödinger Equation and Heisenberg Ferromagnet Equation, TMF (Theor. \& Math. Phys.) 38 (1979) 26-35.

[26] Zakharov V. and Konopelchenko B., On the Theory of Recursion Operator, Comm. Math. Phys. 94 (1985) 483-510.

Alexandar B. Yanovski

Department of Mathematics

and Applied Mathematics

University of Cape Town

7700 Rondebosch, Cape Town

SOUTH AFRICA

E-mail address: Alexandar. Ianovskyeuct.ac.za 